\title{
Do partial cuts create forest complexity? A new approach to measuring the complexity of forest patterns using photographs and the mean information gain
}

\author{
by Isabelle Witté ${ }^{1, *}$, Daniel Kneeshaw ${ }^{1}$ and Christian Messier ${ }^{1}$
}

\begin{abstract}
Forest management generally simplifies forest structure and composition with some negative impacts in terms of biodiversity and resilience. Thus, maintaining structural complexity is increasingly cited as an objective of sustainable forest management. Different initiatives have been proposed to use partial cuts to increase the complexity of forests. Using "the length of description" of forest patterns as a novel measure of complexity in forests, the effects of two intensities of partial cuts were compared to those found in 34-year-old secondary forests and 86-year-old primary (post-fire) forests. Our results show that partial cuts increase the complexity of forest patterns as compared to mature and secondary forests.
\end{abstract}

Keywords: complexity, partial cuts, intermediate disturbance hypothesis, patterns, canopy openness, digital photographs, ecosystem management

\section{RÉSUMÉ}

Laménagement forestier contribue à simplifier la structure et la composition forestière. En conséquence, le maintien de la complexité structurelle est de plus en plus cité comme objectif de l'aménagement durable des forêts. Différentes initiatives proposent d'utiliser les coupes partielles de manière à augmenter la complexité des forêts. En utilisant la longueur de la description des patrons forestiers comme un nouvel indicateur de complexité des forêts, nous avons comparé les effets de deux intensités de coupes partielles sur la complexité des patrons forestiers, aux complexités mesurées dans des forêts fermées. Nos résultats montrent que les coupes partielles permettent d’augmenter la complexité des patrons forestiers par rapport aux forêts matures et secondaires.

Mots clés : complexité, coupes partielles, hypothèse de la perturbation intermédiaire, patrons, ouverture de la canopée, photographie numérique, aménagement écosystémique

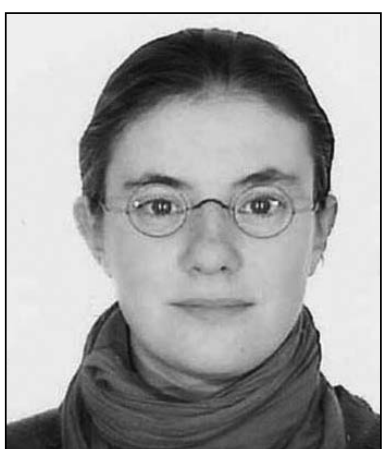

Isabelle Witté

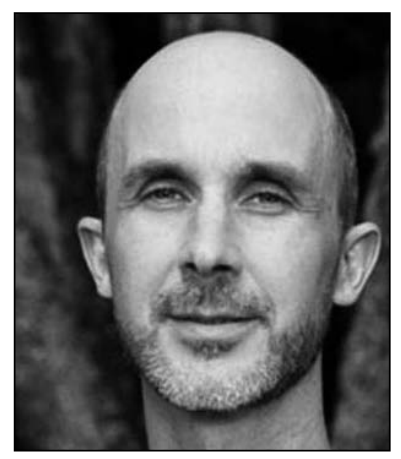

Daniel Kneeshaw

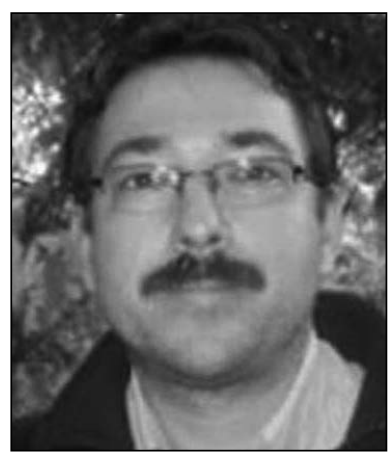

Christian Messier and pushed policy-makers and forest managers to adopt new practices aimed at maintaining or favouring the natural complexity of the forest. Managing for complexity (Puettmann et al. 2009) has therefore recently emerged as a new objective in forest management. The integration of complexity into forest management would involve designing harvest operations that maintain or enhance the capacity of the systems to evolve and adapt to changing conditions. Operationally, managing for complexity involves conserving or recreating complex patterns in the distribution of forests structures and objects (Heinimann 2010). This complexity of patterns guarantees the diversity of associations and spatial configurations between objects and should guarantee the conservation of processes (Turner 1989). We base our definition

${ }^{1}$ Centre détude de la Forêt, Département des Sciences Biologiques, Université du Québec à Montréal, C.P. 8888, Succ. Centre-Ville, Montréal H3C 3P8. ${ }^{\star}$ Corresponding author. E-mail: isa.witte@gmail.com 
of the complexity of forest patterns on Kolmogorov's complexity (Li and Vitányi 1994), such that a compositionally diverse, spatially heterogeneous forest will be complex as it will require a large amount of information to describe all species, their positions and their variation in size to fully conceptualize the system. In contrast, a perfectly regular mono-specific forest may be approximated by describing only the spacing between the stems, which are all of the same species and similar size class ( $\mathrm{Li}$ and Vitányi 1994, Proulx and Parrott 2008).

Partial cutting has recently been proposed as a method of increasing the proportion of uneven-aged and structurally heterogeneous stands (Messier et al. 2013). This approach, by creating gaps in closed even-aged forests or by leaving structural legacies in harvested plots, may enhance the structural complexity of the systems earlier in the regeneration process, and thus the functions and spatial patterns associated with older, more complex forests should be restored faster and more efficiently (Drever et al. 2006). Disturbances can be considered to be part of the cycle of resilience of forests as they allow for a reorganisation of the system and thus for an adaptation to new environmental conditions (Holling 2004). In the case of closed-canopy, even-aged forests, harvesting can be used as an opportunity to increase the adaptive capacity of forest systems (Gunderson 2000). If the complexity of forest patterns is an indicator of forest organisation and adaptability (i.e., it reflects the diversity of associations and processes), then, it may be possible to use partial cuts to increase resilience by increasing the complexity of spatial patterns.

Moreover, the intermediate disturbance hypothesis (IDH) (Connell 1978, Denslow 1985) proposes that species diversity should be highest when moderate disturbances occur in an ecosystem. Moderate disturbances should not only allow the coexistence of species associated with severe disturbances and of species associated with light infrequent disturbances, but should also limit the abundance of dominant competitive species and thus allow an increase in the proportion of less common species (Connell 1978, Denslow 1985). As a potential for reorganization and for the introduction of new species and new structures, we extend the concept and propose that intermediate disturbances could have a positive effect on the complexity of forest patterns by diversifying the possible spatial associations between forest objects (Proulx and Parrott 2008).

Operational indices of complexity that can be used to test the effect of forest management on forest complexity and thus the applicability of the intermediate disturbance hypothesis are, however, still rare in forest ecology. Few previous studies have investigated the effects of new harvest strategies on the complexity of forest patterns. Instead, earlier studies have looked at the relationship between species and structural diversity. Years of study have thus led to knowledge of the response of specific structures such as living trees (Crow et al. 2002, Brais et al. 2004, Saunders and Wagner 2008), dead wood, (McGee et al. 1999) and understory plants (Roberts 2007) to different types of harvesting. On the other hand, the effects of partial cuts on the spatial distribution of structure and habitat heterogeneity have also been studied for different animal taxa (Suzuki and Hayes 2003, Loehle et al. 2005, AguilarAmuchastegui and Henebry 2007). Considering this early work, complex forests can be defined as diverse and structurally heterogeneous systems that provide a large variety of habitats for plants and animals. As a consequence, much research has attempted to measure the complexity of forests at different scales as a combination of indices of both diversity and heterogeneity (Van Den Meersschaut and Vandekerkhove 1998, Staudhammer and LeMay 2001). These indices are generally very thorough in their description of the structural composition of forest plots, accounting for several compartments (e.g., dead trees, lying trees, understory structure, canopy) and for the variability of that composition (Van Den Meersschaut and Vandekerkhove, 1998), but are actually often lacking a description of the spatial structure of the system. Hence most structural indices give a complete idea of the diversity of the structures (e.g., average tree diameters, layering, age classes) in forest plots, but lack a description of the spatial associations between objects. Several attempts have however been made to capture the spatial components of forest structures, and to represent the association of the different objects (Barbosa Lima et al. 2006, Pommerening 2006, Wiegand et al. 2006, Grabarnik and Särkkä 2009, Kretzschmar et al. 2010), with interesting outcomes regarding the importance of pattern characterization for the understanding of forest processes. However, since the dominant approach to spatial patterns in ecology is still based on point pattern analysis (aggregation clumping or regularity indices based solely on a two-dimensional position of the objects), the amount of data required to achieve a representation of the complexity of pattern accounting for both the diversity of structures and their spatial distribution tends to be overwhelming. A relevant measure of complexity in forest stands should therefore include both the diversity of structures and their spatial distribution, while at the same time remaining operationally measurable.

Different attempts have been made to develop such an index using patterns that emerge from the juxtaposition of several objects. These emergent patterns can be the visible results of the spatial associations between forest objects (e.g., trees forming forest patches in a landscape, forest edges defined by individuals, canopy gaps delineated by the foliage) and can be studied as objects, by defining their shapes (e.g., regularity, fractal dimensions) (Sugihara and M. May 1990, Hagerhall et al. 2004), dimensions (e.g., surface area, perimeter, richness of components) and spatial characteristics (aggregation, spatial autocorrelation) (Zenner and Hibbs 2000, Marsden et al. 2002). The characterization of emergent patterns as complexity indicators is compatible with the use of remote sensing methods such as digital photographs (Marsden et al. 2002), aerial photographs (Hagerhall et al. 2004) or LIDAR (Vepakomma et al. 2008, Kane et al. 2011).

Here we use a new approach to the characterization of patterns in digital photographs based on the distribution of pixels. Rather than defining shapes from the delineation of objects (trees or canopy openings), the pixels are used as individual objects. The spatial distribution of the colours of pixels and the information that they entail is used as a representation of the spatial associations between forest objects such as trees, canopy opening, and understory plants.

We expect that intense disturbances, such as clearcuts, that tend to leave only bare soils and open skies, will have relatively regular spatial patterns on photographs. On the other hand, closed forests should also appear relatively regular as they are formed of multiple layers of vegetation creating a uniform green pattern until the forest starts to break up and create more complex patterns of openings (canopy gaps) within the closed forest. We expect that intermediate disturbances that allow interfaces to appear between closed forests and open areas would increase the complexity of the resulting patterns. However, at the scale of forest plots, partial cuts could also create openings that are 
contrasted with the surrounding intact forest and simply create a juxtaposition of two relatively simple patterns. In this case, the technique of partial cutting (distribution of openings and tracks) could be of greater importance for the resulting complexity of the forest plot than the amount of tree removal.

In this paper, we test a new approach to measuring complexity by comparing the spatial patterns emerging from different management histories (former clearcuts, maturing forests and partial cuts). More specifically, we test whether complexity is greater in forests that have been subjected to an intermediate disturbance such as partial cuts compared to undisturbed or young secondary forests.

\section{Methods}

Study area

Study sites were located in the ecosystem management area of the TRIAD project near the town of La Tuque $\left(47^{\circ} 26^{\prime} 00^{\prime} \mathrm{N}\right.$, $\left.72^{\circ} 47^{\prime} 00^{\prime} \mathrm{W}\right)$ in central Québec. The TRIAD project is based on the use of three different management objectives (conservation, ecosystem management and intensive management) within the 0.86 million ha forest management unit. The different land uses are spatially distributed in order to optimize the associations between these different land uses at different scales (Tittler et al. 2001, Messier et al. 2009). Each component of the TRIAD zone has different but complementary objectives: 1) the conservation zone aims at allowing forest aging and colonization by late-succession species, 2) the intensive forestry zone is based on the plantation of fast-growing species and aims at partially compensating the loss of wood production caused by the conservation area, 3) the ecosystem management zone involves different intensities of harvest methods ranging from traditional clearcuts to partial cutting. The partial cuts in the ecosystem management zones of the TRIAD project were designed with the objective of increasing the proportion of uneven-aged and heterogeneous stands.

\section{Sampling}

All study sites were included within a $2.5-\mathrm{km}$ radius area at the northern limit of the mixed-wood conifer and hardwood forest in Haute-Mauricie, Québec. The study forests are dominated by balsam fir (Abies balsamea [L.] Mill.), white spruce (Picea glauca [Moench] Voss), red maple (Acer rubrum L.) and yellow birch (Betula alleghaniensis Britt.). We sampled four different forest treatments corresponding to different forest histories. All forest treatments originated from a major fire in 1923 and were then managed differently. Mature forests (MF) were left untouched, secondary forests (SF) were clearcut during the 1970s and were untouched since. Partial-cut treatments (PC50 and PC35) were both partially harvested during the winter of 2007-08.

Both partially cut forests were harvested with the same basic technique. A 5-m wide temporary track was clearcut, and from that track two $7-\mathrm{m}$ wide strips were thinned to $\sim 50 \%$ of their basal areas. Between these harvested strips retentions areas of respectively $5 \mathrm{~m}$ and $19 \mathrm{~m}$ for PC50 and PC35 were left uncut in order to attain a total $50 \%$ or $35 \%$ basal area removal (see Fig. 1 for an illustration and explanation of the harvest design).

In each treatment we inventoried and photographed three $40 \times 40-\mathrm{m}$ replicate plots during the summer of 2008 (PC35 and PC50) and 2009 (MF and SF).

\section{Photographs sampling}

Sixteen $10 \times 10$ - $\mathrm{m}$ sub-plots were photographed in each of the 12 ( 4 treatments $\times 3$ repetitions) $40 \times 40-\mathrm{m}$ plots (Fig. $2 \mathrm{a}$ ). As two of the forest treatments involve partially cut strips, and in order to avoid the directional effects of the vegetation structure that resulted from that harvesting design, each subplot was photographed in two perpendicular directions (see Fig. 2b).

Photographs were taken at each of the photo-points from three different angles, with the camera pointing to three different layers of the forest structure (see Fig. $2 \mathrm{c}$ and Table 1 for the camera settings). Canopy photographs were taken with $\mathrm{a}+45^{\circ}$ inclination of the camera; mid-forest layer photographs with a $0^{\circ}$ inclination and finally the understory photographs with a $-30^{\circ}$ camera inclination (see Fig. 3 for examples of the resulting photographs).

This design results in 16 pairs of photographs for each of the twelve $40 \times 40$ - $\mathrm{m}$ plots and each layer of vegetation (Canopy, Mid-forest and Understory), each pair corresponding to a $10 \times 10-\mathrm{m}$ subplot. a

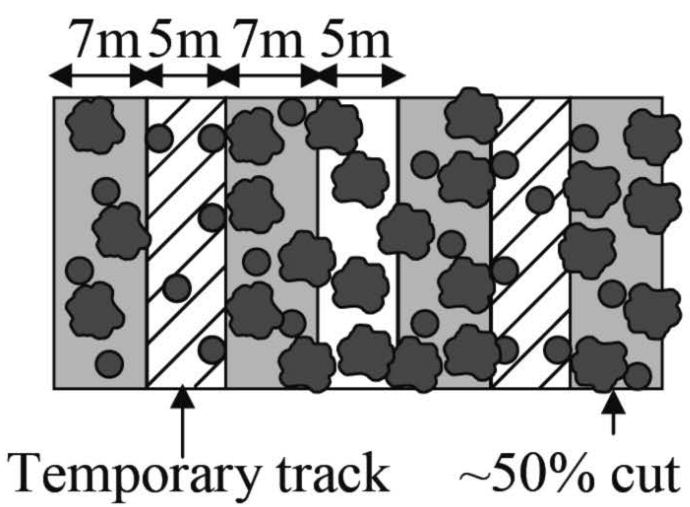

$\mathrm{b}$

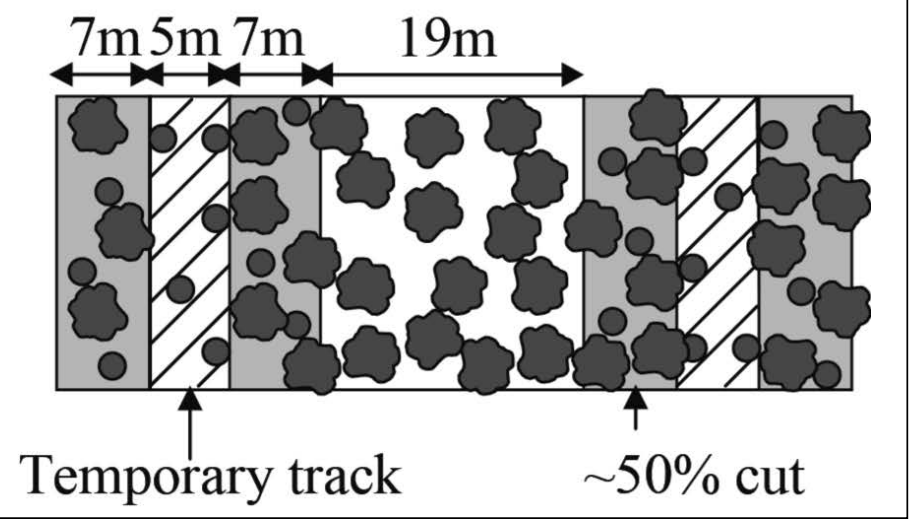

Fig. 1. Harvest designs for (a) 50\% partial cut (PC50\%) and (b) 35\% partial cut (PC35\%). The partial cuts are organized around 5-m-wide skid temporary tracks that are clearcut. About $50 \%$ of the basal area is harvested from two 7-m-wide bands (grey bands) on each side of the skid trails. These cut blocks are separated by uncut bands (white bands) of varying width: 5 m in PC50\% (a) and 19 m in PC35\% (b). 


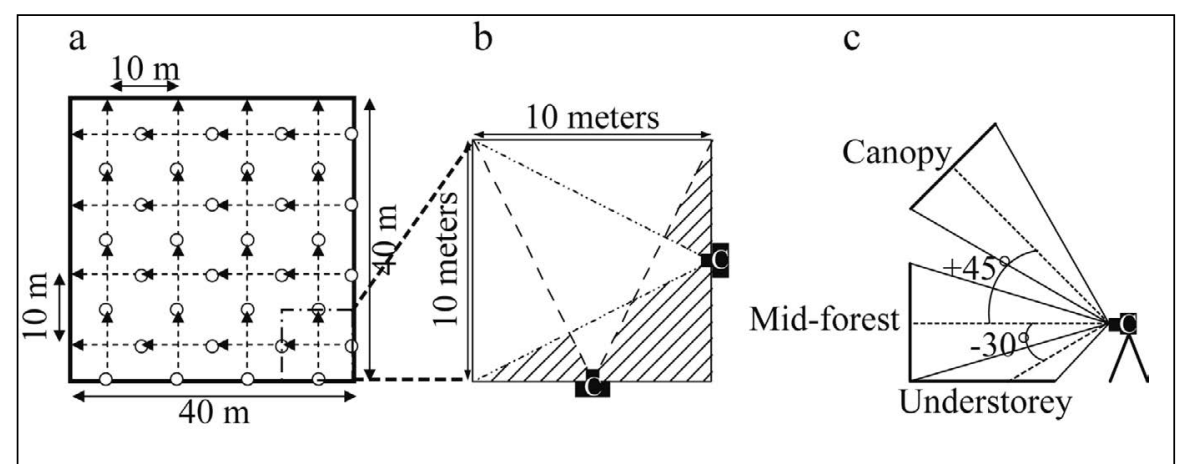

Fig. 2. a) Schematic map of a 40x40-m plots with the different positions of the camera (open circles) and the directions of the photographs (dashed arrows). b) Schematic representation of photographic sampling in 10×10-m subplots. Hatching represents the dead angles of the cameras. c) Angles of the camera for the three layers of vegetation (canopy, midforest, understory]. Dashed lines represent the centre of the photographs with the angles of inclination for the camera.

All photographs were taken in similar light conditions under overcast sky in order to limit the influence of light variations and direct sunlight (shadows, over exposure).

\section{Measuring complexity: Mean information gain (MIG)}

The description of the complexity of vertical patterns in forests is generally summarized by variation in canopy heights (Zenner and Hibbs 2000) or by visual estimates of variation in the density

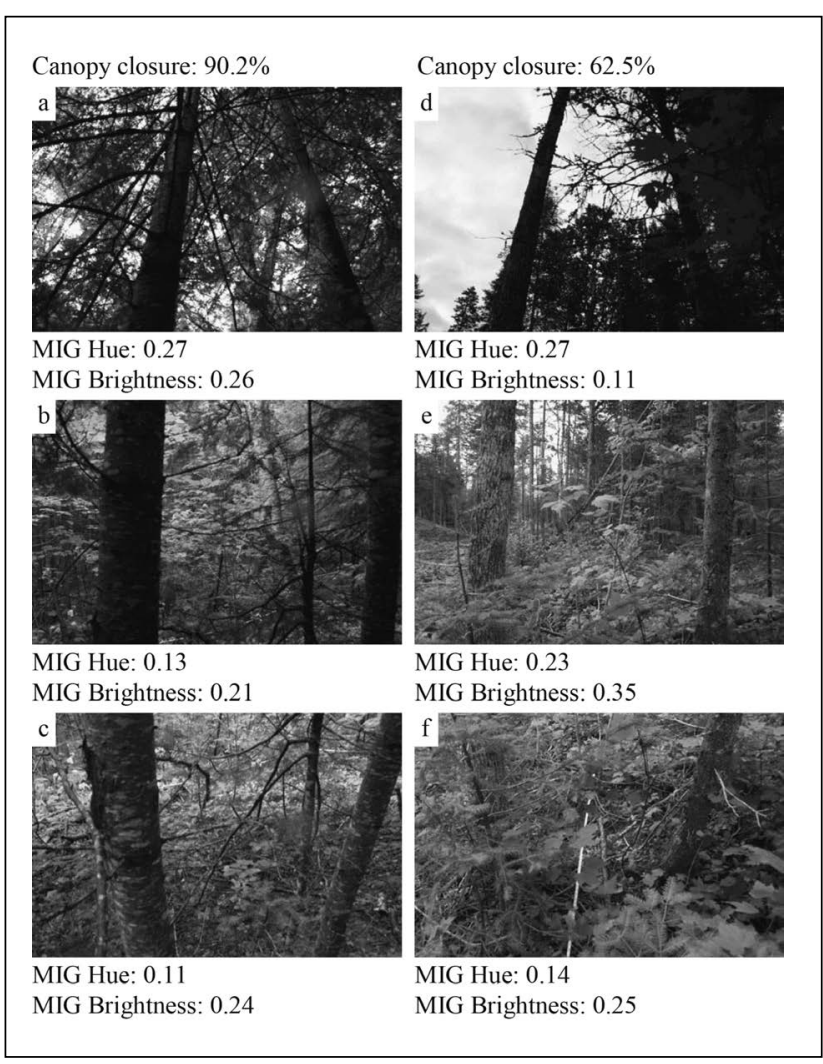

Fig. 3. Examples of photographs from the 3 forest layers in Haute-Mauricie, Québec. Canopy (a and d), Mid-forest (b and e), Understory(c and f), in maturing forests (MF, a, b and c) and $50 \%$ partial cut (PC50\%, d, e and f). of vegetation layers (Neumann and Starlinger 2001, McElhinny et al. 2005). Here we chose to implement a method based on the measure of the mean information gain (MIG) in photographs, developed by Proulx and Parrot (2008). The method consists of measuring the disorder of patterns in digital photographs. Digital photographs provide an intensive sampling of all visible objects that constitute the vertical structure of the forest stands. The values of each pixel represent the colour characteristics and hence the observable differences between the objects that were photographed. The spatial associations of pixels in the resulting $2 \mathrm{D}$ pictures reproduce visible patterns of the spatial distribution of these objects. The complexity of the patterns can therefore be measured directly on the matrices of pixel values in the photos. This sampling method also provides a repeatable and potentially automatable method to monitor the complexity of forest systems over large areas or in time.

The MIG is a measure of the complexity of patterns based on Kolmogorov information complexity (Li and Vitányi 1994). In simple words, the MIG allows one to measure the disorder of a pattern by quantifying the length of its description. A regular pattern such as a checkerboard (see Fig. 4a) for example, is identified as a simple ordered pattern as its description only requires the dimensions of the pattern $(8 \times 8$ pixels $)$ and the identity and sequence of the pixels (white-black). On the other hand, in order to describe a completely random pattern with the same level of precision, each individual pixel must be described as its identity and position cannot be predicted from its neighbours. Random patterns are therefore described as maximally disordered. MIG values range between 0 and 1 ; regular patterns are characterized by low values and random patterns by high values. Complex ecological patterns are considered to be neither completely regular nor completely random (see Fig. 4b) (Andrienko et al. 2000, Parrott 2005, Dale et al. 2007). In previous research by Proulx and Parrot (2008), the most complex patterns were shown to be represented by intermediate values of MIG.

Table 1. Camera settings (see Proulx and Parrott [2008] for more explanation about the choices behind these settings]

\section{Setting}

Camera

Focal length

Aperture diameter

Focus distance

Tripod's head above ground

Depth of field (DF)

Exposure mode

Time window for shooting

Visual obstruction $<$ DF

White balance mode

Resolution
Canon EOS Rebel XTI 400D

$18 \mathrm{~mm}$

$6.3 \mathrm{~mm}$

$10 \mathrm{~m}$

$1.3 \mathrm{~m}$

$2 \mathrm{~m}$ - infinity

Aperture priority

7h00 - 16h00

Avoided

Natural light

$1288 \times 1936$ pixels 


\section{MIG computing}

Traditionally, digital cameras record pictures in RGB (Red Green Blue) format, but for a more direct link to the actual colour of objects and effects of light, our photographs were converted to HSB images (Hue Saturation Brightness) following the method proposed by Smith (1978). Each pixel in the image thus bears three values respectively defining its colour (hue), the intensity of the colour (saturation) and the quantity of light it received (brightness).

The values of pixels in the HSB domain are originally continuous numerical values, but were clustered into a smaller number of classes of values for the computing of MIG. This clustering of values is necessary as with continuous values, each pixel can potentially be unique, and can thus result in false random patterns. The final number of classes of values $(\mathrm{N})$ is limited by the resolution of the images and by the size of the associations of pixels ( $n$ ) considered in the computing of MIG. Proulx and Parrot (2008) conducted sensitivity tests of the effects of variations in light and pixel resolution on MIG and subsequently prescribed optimum values, which we use in this paper (see Table 1). The ratio of the resolution to the maximum number of possible combinations between $n$ pixels must remain $>100$ in order to exclude the possibility of falserandom patterns (see eq. 1).

$$
\text { [1] } \frac{\text { Resolution }}{N^{n}}>100
$$

In our case, for pictures of resolution $=1288 \times 1936$ pixels and $n=2 \times 2$ pixels, we reduced the number of classes for each band to $N=10$ classes (for a ratio $\sim 250$ ).

Once the image is converted to HSB and the number of classes of values is reduced, the three data matrices of hue, saturation and brightness values can be used to calculate the complexity of the patterns of pixels. The MIG is computed based on the diversity of the spatial associations of $2 \times 2$ pixels for each band (HSB) of the photographs using eq. 2 (Andrienko et al. 2000, Proulx and Parrott 2008):

$$
\text { [2] } \quad \text { MIG }=\frac{\left.\mathrm{H}[\chi]-\mathrm{H}^{\gamma}\right]}{\log N^{4}-\log N^{1}}
$$

$N^{4}$ is the maximum number of $2 \times 2$ combinations of pixel values, $\mathrm{H}[\chi]$ is the Shannon diversity of $2 \times 2$ pixels associations (see eq. 3), and $\mathrm{H}[\gamma]$ is the Shannon diversity of individual pixel values in the image (eq. 4) (see also Fig. 5 for an illustration of patterns and their associated values of diversity for pixel associations and individuals).

$$
\text { [3] } \mathrm{H}[\chi]=-\sum_{\mathrm{i}=1}^{N^{4}} \mathrm{p}(\chi \iota) \log \mathrm{p}\left(\chi_{\mathrm{i}}\right)
$$

$\mathrm{p}(\chi \mathrm{i})$ is the probability of finding a specific $2 \times 2$ combination $\chi^{\mathrm{i}}$ of pixel values in the image

$$
\text { [4] } \mathrm{H}\left[\gamma^{\gamma}\right]=-\sum_{\mathrm{i}=1}^{N} \mathrm{p}\left(\gamma^{\prime}\right) \log \mathrm{p}\left(\gamma_{\mathrm{i}}\right)
$$

$p(\gamma \mathrm{i})$ is the probability of observing a pixel value $\gamma \mathrm{i}$ independently of its location in the image.

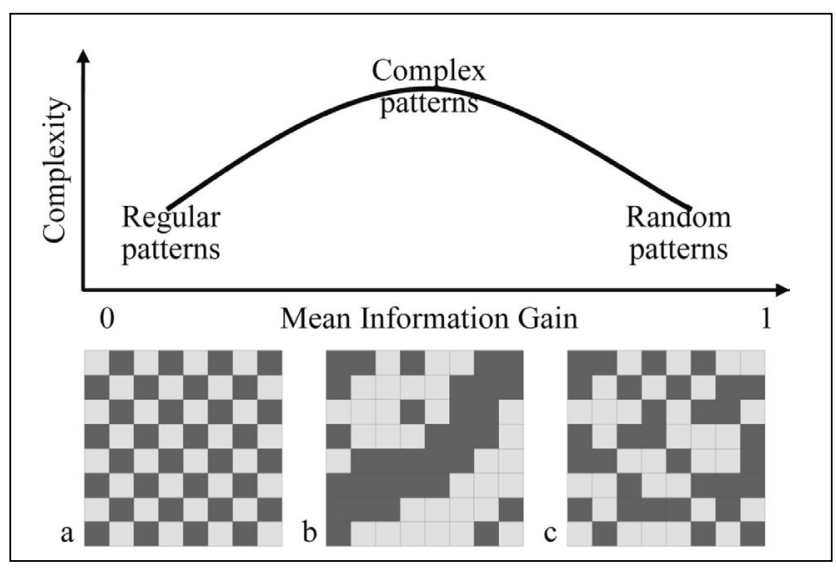

Fig. 4. Convex relationship between the mean information gain (a measure of complexity based on the length of the description of the pattern) and the complexity of spatial patterns. a) Regular pattern, b) complex pattern, c) random pattern. Note that although the three patterns $(a, b$ and $c$ ) have the same pixel composition (32 white and 32 grey pixels ) their MIG value varies as a function of the complexity of the spatial pattern (see text for details).

a
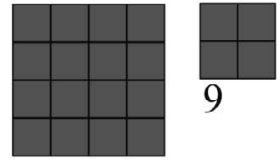

$\mathrm{b}$
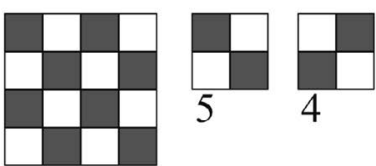

c
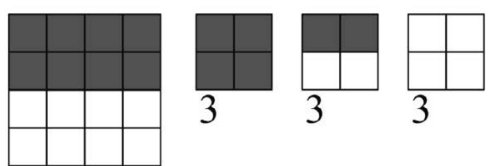

d
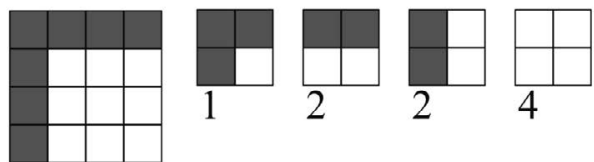

e
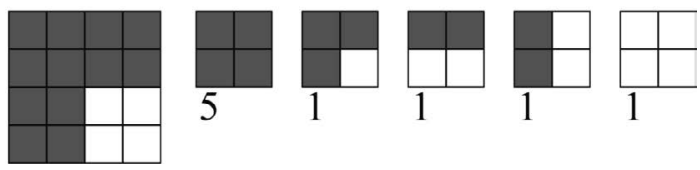

Fig. 5. Examples of patterns created with $N=2$ classes of pixels values (black or white) with increasing complexities (MIG values: $a=0, b=0, c=0.19, d=0.28, e=0.35$ ) and the list of $2 \times 2$ pixel associations that compose them, the number below each pixel association is its number of occurrence in the pattern. The resulting diversities of spatial associations $\mathrm{H}[\chi]$ are: $\mathrm{a}=0$, $b=0.69, c=1.10, d=1.27, e=1.30$ and the diversities of individual pixel values $H[\gamma]$ : $a=0, b=0.69, c=0.69, d=0.69$, $\mathrm{e}=0.56$. 
MIG indices are computed for each photograph separately and then the MIG values are averaged across the pairs of photographs corresponding to each $10 \times 10-\mathrm{m}$ subplot. This resulted in 48 values of MIG for each combination of layer (Canopy, Midforest and Understory) and HSB band in each forest treatment. All computing for MIG measurements of the digital photos was done using MATLAB (V 7.6, 2008).

The values of MIG in the hue band show the complexity of spatial associations between the colours in the photographs and are therefore related to the potential for spatial associations between objects of different nature (e.g., white sky and green leaves, leaves or trunks of different shades, green vegetation and brownish bare soil). The interpretation of Saturation patterns is based on the interactions between light and colour of forest objects. Hence, low values of Saturation can be achieved either because the colour of the objects is actually dull, or because the object is in plain light or in the shade as both bright and insufficient light tend to fade the colour of objects. In the case of forests, the scenes are generally dark, and the apparent saturation of colours increases with brightness. The patterns in the brightness band represent the complexity in the distribution of light and its interactions (reflection, absorption) with the objects in the photographs (e.g., trunks, leaves, soil). As patterns in the saturation and brightness bands are closely correlated and as the patterns of brightness are more directly related to ecologically relevant characteristics of forest objects, we chose to only present the results for the complexity of patterns in the hue and brightness bands.

\section{Canopy closure}

The two photographs of the canopy layer in each $10 \times 10-\mathrm{m}$ subplot were also used to measure canopy closure (see Fig. 2c). These photographs were manually converted to black and white by selecting a threshold level that allowed the distinction between sky and vegetation. The closure of the canopy for each image was defined as the percentage of black pixels in the image. The final value of canopy closure for each $10 \times 10-\mathrm{m}$ subplot is the average of the canopy closure measured from the two original photos.

\section{Data analysis}

The MIG values were compared among the treatments using an analysis of variance (ANOVA) with the $40 \times 40-\mathrm{m}$ repetition plots embedded in forest treatments as fixed factors. Tukey's test was used as a post-hoc test to hierarchically differentiate the treatments ( $\mathrm{R}$ 2.12.2, package: stats) ( $\mathrm{R}$ Development Core Team 2010). Each treatment was therefore represented by 48 values resulting from the three repetition plots and $16 \mathrm{MIG}$ values in each plot.

The relationships between the 192 values (48 values from each of the four treatments) of canopy closure and the complexity of patterns were computed using mixed-effect models with the $40 \times 40-\mathrm{m}$ plots included as random factor (R 2.12.2, packages: stats, nlme, AICcmodavg) (R Development Core Team 2010, Mazerolle 2011, Pinheiro et al. 2011). Linear and second-degree polynomial models for the relationship between forest closure and complexity were tested for both data from hue and brightness bands and for all the vegetation layers and compared using ANOVAs to select the best fit. The squared correlation coefficients between the observed data and the models were also computed in order to present an estimate for the fit of the models.
Fig. 6. Hue (left) and brightness (right) bands in four forest treatments for each of the layers of vegetation ( $a$ and $d$ : canopy, $b$ and e: mid-forest layer, $c$ and f: understory) in forests of Haute-Mauricie, Québec. P-values for ANOVA tests between forest treatments are all $<0.01$ Intervals represent the standard deviations for each sample; Different letters show significant differences as determined by Tukey post-hoc tests on forest treatments with $\alpha=0.05$.

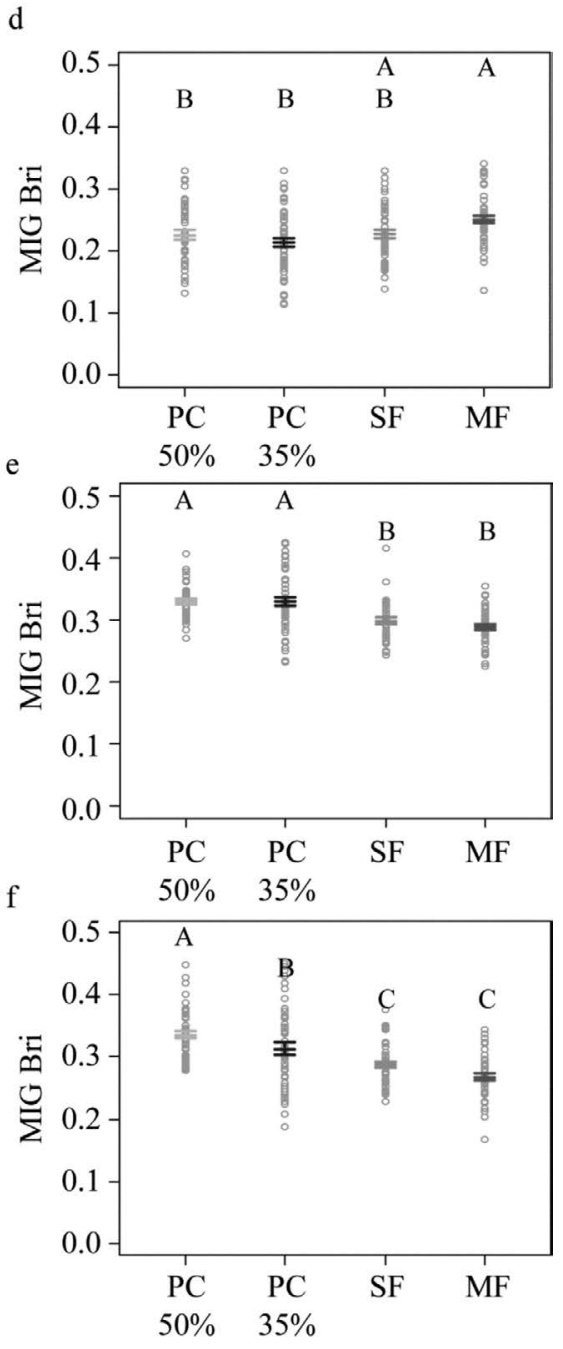

MAY/JUNE 2013, VOL. 89, № 3 - THE FORESTRY CHRONICLE 


\section{Results \\ Range of complexity of patterns in managed boreal mixed- wood forests}

The values of MIG in our data set ranged from 0.08 to 0.45 , and thus ranged from very regular (MIG tends to 0 ) to complex (MIG tends to 0.5). We therefore considered our gradients of MIG values directly as gradients of complexity with high MIG values representing complex patterns and low MIG values representing regular patterns.

\section{Effects of forest treatments on the complexity of patterns in the hue band}

For the canopy layer, both partial-cut forest treatments (PC50\% and PC35\%) had significantly higher MIG values than the mature forest (all p-values $<0.001$ for the hue band, see Fig. $6 a$ ). Similarly for both lower canopy layers (Fig. 6b and c), MIG values were highest for PC50\% and lowest for the closed forests (MF and SF). Mature and secondary forests were found to have consistently similar complexity of patterns for all layers (Fig. 6a, b and c). Also, MIG values were clearly higher for the uppercanopy layer compared to the two lower canopy layers. We did not undertake any statistical analysis among canopy layers because the scope and depth of the photographs taken at different heights (canopy, mid-forest, and understory) were very different. Such comparison between layers should be tested in the future.

\section{Effects of forest treatments on the complexity of patterns in the brightness band}

In the canopy layer, the lowest MIG value was found for the PC $35 \%$ forest treatment and the highest for the mature forest treatment (all p-values $\leq 0.001$ for the brightness band, Fig. $6 \mathrm{~d})$. However, for both lower layers (mid-forest and understory), the tendency was to observe decreasing MIG values from the most open stand (PC50\%) to the most closed (MF) (Fig. 6e and f). Contrary to MIG values for hue patterns, brightness patterns tend to be more complex in the lower layers than in the canopy layer.

\section{Canopy closure and the complexity of patterns}

Mean canopy closures for the different treatments were $65 \%$ for PC50\% (range from $26 \%$ to $85 \%$ ), $68 \%$ in $\mathrm{PC} 35 \%$, (38\% to $90 \%)$, $86 \%$ in SF (76\% to $93 \%)$ and $89 \%$ in $\mathrm{MF}(80 \%$ to $96 \%)$. The relationships between complexity (as measured by MIG) and canopy closure for the canopy layer were convex for patterns of both hue and brightness $\left(R^{2}=0.27\right.$ for hue patterns and $R^{2}=0.24$ for brightness) (Fig. 7a and b). This relationship shows that the complexity of hue and brightness patterns reaches a maximum respectively around $60 \%$ to $70 \%$ and $75 \%$ to $85 \%$ of canopy closure. This suggests that the complexity of canopy photos is related to the proportion of sky visible through the foliage, with a maximum for slightly open canopies.

In the two lower layers (mid-forest and understory Fig. 7c to $\mathrm{f}$ ), the relationships between complexity and canopy closure were generally negative with complexity decreasing with increasing canopy closure. Patterns in the hue band appear less complex in closed forests, which support our results among forest treatments. This indicates that the associations of hue values between forest objects are more regular in closed forests and that the same associations of colours are repeated in the image. In the same way, patterns of brightness values are more regular in closed forests with less diversity in the associations of light intensities.
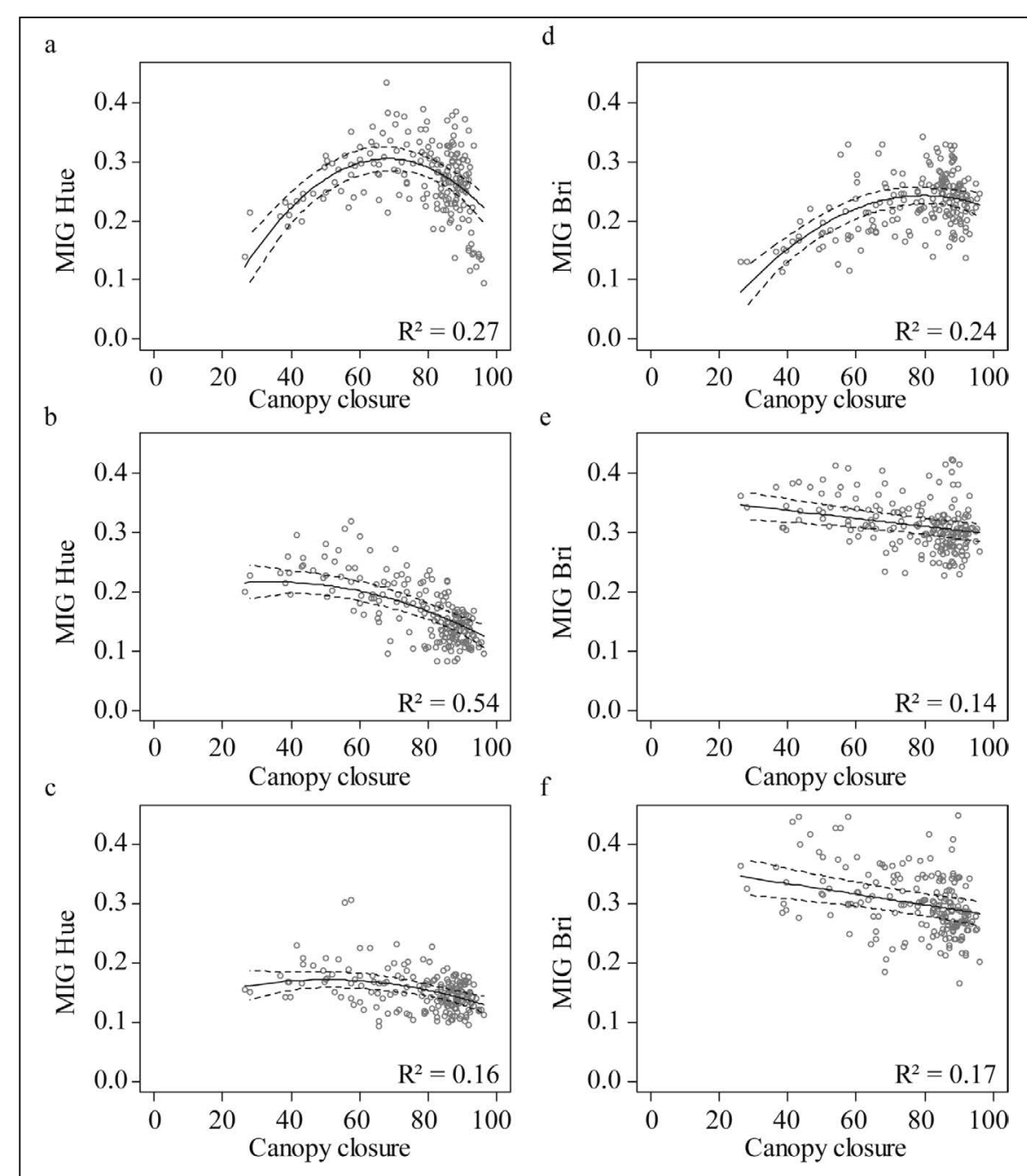

Fig. 7. Relationships between canopy closure and MIG values (solid lines) for the hue (left) and brightness (right) bands for all forest treatments together for each layer: a and b: canopy, c and d: mid-forest layer, e and f: understory. Dashed lines represent the 95\% confidence interval for the model. The $R^{R}$ are the squared correlation coefficients between the observed and the modelled data. 


\section{Discussion}

The complexity of patterns as measured by MIG from the digital photos of forests comes from the complexity of assemblages between forest objects and the visual contrasts between these objects. The forest stands identified as complex by the MIG are thus those that allow a variety of different associations to occur between different forest objects and light intensities (Proulx and Parrott 2008).

Over the relatively small range of forest treatments investigated in this study, partial-cut stands had higher levels of complexity of patterns than closed-forest stands. Also, the relationship between canopy openness and the complexity of patterns indicated that both the highest and lowest intensities of disturbance can induce a decrease in the complexity of patterns (Fig. 7a, b, c and e). Although a number of different factors could influence the spatial structure and diversity of forest plots, we focused on the effects of silvicultural treatments by choosing forests similar in structure and composition pre-treatment for our comparison. However, it is clear that different management histories influence more than the canopy openness, and have effects on the stage of stand development of the stand as well as on its species composition. As MIG measures the complexity of the pattern in photographs that integrates all structural variations, our observations are bound to integrate the influence of the whole history of the forest plots and not only the latest management operations (the different partial cuts and clearcuts). However, the consistent similarities between MIG values for mature and secondary forests (MF and $\mathrm{SF}$ ) and the differences between partially cut forests and closed forests (PC50 and PC35 vs. SF and MF) (see Fig. 6), supports the conclusion that the removal of trees and consequent canopy opening are important in determining the complexity of patterns. However, further work examining the effects of stand development stages or variations in species composition would be valuable in order to understand pattern complexity as measured by MIG in digital photographs.

These findings suggest that partial harvests increase the complexity of patterns in forests and that the effects of these moderate disturbances (i.e., between clearcut and no harvesting) support the "intermediate disturbance hypothesis" in terms of its application to complexity.

\section{How to interpret the complexity of forest patterns measured in photographs}

Understanding the complexity of forest pattern measured by the MIG depends on understanding the relationships between the MIG and more traditional features of forest structure. First, the complexity of patterns measured by MIG is not directly related to species diversity. The diversity of species only affects the complexity of patterns if the species are visually different (e.g., in shape, spatial distribution of biomass, colour, reflection or absorption of light). For example, in our canopy photos, the juxtaposition of two species with foliage of similar shades of green will not increase the complexity of forest pattern in the images. On the other hand, the juxtaposition of the sky with leaves (different colours) or different superposition of leaves (different light intensities) would lead to higher diversity of patterns and thus to higher overall complexity. The complexity of patterns measured by MIG may thus be higher in canopies with lower species diversity, but more leaf layering, than in species-diverse canopies but with no layering (see the visual effect of leaf layering in Fig. 3a). Also, objects that are visually different, but that are clearly separated in the photographs, will tend to produce simple patterns when measured with the MIG index as the resulting pattern will be a juxtaposition of uniform areas (e.g., blue sky over brown soil). Hence, complex patterns emerge from the spatial mixing of different objects and not only from their presence.

The relationship between the complexity of patterns measured by the MIG and light is somewhat more complicated. The MIG obtained on the brightness band measures the interaction between light and objects in the forest. Technically, the patterns of light that appear in photographs are only the results of the patterns created by the vegetation. In photographs the light only reveals the position of the objects by being either reflected by their surfaces or obstructed by them. From an ecological point of view however, light and the patterns it creates are determinants for several processes from plant regeneration and growth to decomposition and soil chemistry (Endler 1993, Denslow and Guzman 2000, Beaudet et al. 2004). Thus, the complex patterns in the brightness band that emerge from the irregular distributions of forest objects in space may also be representative of the occurrence of various plant and forest processes that are dependent on light distributions.

\section{Partial cuts allow original associations of forest objects}

The removal of some trees in otherwise dense and closed forests by partial cuts creates new forest patterns (McElhinny et al. 2005). In this study, partial tree harvesting increased the diversity of visible forest objects by mechanically reducing the presence of trees in the photographs. In the mid-forest and understory layers, the more complex patterns emerging after partial cuts are therefore caused by original associations between objects from different forest strata (soil juxtaposed with tree trunks, understory plants associated with the sky due to canopy openings) (Proulx and Parrott 2008). The increased complexity of hue patterns in the recently partially cut forests emerged from the reduced density of trees and understory plants in the pictures (see the example photographs in Fig. 3). The pictures taken in the partial cuts thus include patterns with features from both closed forests (uniform green screens of vegetation) and open systems (sky, bare soil and vegetation mixed at all height of the image).

The complexity of patterns in the brightness band is driven by the same type of effects on the structure of forest communities. In the lower layers (mid-forest and understory) the openness of the canopy allows more light to penetrate through the layers of the community (Beaudet and Messier 2002) and therefore allows the juxtaposition of very bright areas with darker spots, but also in the same pictures, more subtle gradients of lights in the more densely vegetated areas (see the mid-forest layer in the partial cuts in Fig. 3e). Also, in the case of intermediate disturbances such as partial cuts, light can reach forest objects from different angles (from the top of the canopy but also laterally through gaps or after being reflected off another surface) therefore increasing the diversity of light intensities on similar objects (Endler 1993). In undisturbed closed forests, light is strongly filtered through the dense canopy and variations in brightness occur progressively (hence regularly) along the vertical gradient of light.

In the canopy layer, however, partial cuts create large contiguous canopy openings and therefore a radical increase of incoming light. The intensity of light in that layer tends to increase the contrasts between bright sky and darker objects 
and thus hides the gradients of hue and brightness in the image (Proulx and Parrott 2008). Hence, in the canopy layer of partial cuts, the patterns do not only appear regular because of the large uniform white areas of sky, but also because all of the objects in the picture tend to appear uniformly black (see Fig. 3d). On the other hand, mature and secondary forests generally have closed canopies that allow a filtering of light, thus creating gradients of brightness values. Moreover, even when canopy gaps occur in mature and secondary forests, they tend to be individually smaller and more heterogeneously distributed in the canopy, thus creating more complex patterns.

\section{Effects of canopy opening on the complexity of patterns among vegetation layers}

The opening of the canopy by itself has a strong effect on the complexity of patterns in the different forest treatments. In the lower vegetation layers (understory and mid forest), the complexity of patterns increases with the opening of the canopy, indicating that the diversity of spatial association between objects increases as the effects of disturbances become more severe (Fig. 7b, e and f). In the canopy, however, the complexity of both hue and brightness patterns drops significantly below 55\% canopy cover (Fig. 7a and b). This indicates that the same intermediate disturbance (gaps created by partial cutting) does not affect the complexity of patterns consistently from the top to the bottom of the canopy. This variation can be explained since the reduction of canopy cover in the canopy layer reveals only the underlying uniform sky patterns (Fig. 3d) whereas in the lowest layers the harvest of foreground trees will always reveal more background elements, thus maintaining a high diversity of object associations. However, with an ever-increasing canopy opening, it is likely that the amount of sky in the background will finally increase and cause a simplification of the patterns (and thus a decrease in MIG values) in the lower layers. Hence, we expect that a sample of forest treatments including a larger representation of forests with open canopies would present a convex relationship between canopy openness and MIG even in the lower vegetation layers.

Further study of our MIG index should also take the shape and distribution of canopy gaps into account as well as their origin (natural or anthropogenic). This will allow examination of how the variations in MIG values respond to the shape of gaps and ultimately if the maximum values of complexity that we observed always occur for the same canopy closure regardless of the origins of the gaps. The increased viewing depth in forests with open canopies could also influence the values of MIG, and could be further studied, perhaps in more controlled conditions.

\section{Complex patterns: Signs of creative reorganization and resilience and confirmation of the intermediate disturbance hypothesis?}

According to our work, and in accordance with the intermediate disturbance hypothesis (Connell 1978, Denslow 1985), the complexity of patterns in partial cuts and in stands with some level of disturbed canopies is higher than in closedundisturbed forests (Fig. 7). As they increase the complexity of patterns, partial cuts can thus be considered to be disturbances that encourage a "creative" reorganisation in several forest layers, which might thus increase forest resilience (Holling 2004, Drever et al. 2006). Partial cuts could help recreate the conditions found in old-growth forests when natural gap dynamics help create new opportunities for stands to increase both structural and compositional diversity.
However, in this study we did not investigate the complexity of patterns found in old-growth forests since our oldest forests were less than 100 years old. It is possible that old-growth forests would show more complex patterns for similar canopy opening than partial cuts, as natural gaps tend to be more heterogeneous in shape, size and spatial distribution. It could, for example, be hypothesized that the decrease in the complexity of patterns in forests with high canopy opening will be more rapid in anthropogenically created openings as they will consist of more contiguous and regularly shaped gaps. In addition, the forest objects in the backgrounds of the photographs that allow original spatial associations in our samples would typically be absent if the cut strips were wider or if the cutting intensity was higher, thus causing simplified patterns.

As a consequence, if the management objectives involve increasing the complexity of patterns, partial cuts could be designed in order to avoid large continuous linear open areas. Similarly, the use of linear skid trails (vs. e.g., zigzag patterns) could be questioned as they create long continuous clearcut areas (Beaudet et al. 2004).

Also, as canopy openness is particularly important for the resulting complexity of patterns (Aakala et al. 2012), specific care should be given to the complexity of the forest canopy before assigning a specific harvest method or intensity of cut. Moreover, at the scale of the entire forest (e.g., on aerial photographs), measures of the MIG of partial cuts such as those that were studied in our work would be expected to identify relatively regular strip-cut patterns. It may therefore be advisable to not apply the same method of cutting everywhere so as to increase the overall complexity of patterns at the forest scale.

Further work including testing the complexity of patterns in more intense disturbances and older forests undergoing gap dynamics as well as at larger forest scales on aerial photographs could help confirm our findings and provide more definitive tests of our hypotheses.

\section{Conclusion}

Using a new method to measure the complexity of patterns in forests, we showed that moderate anthropogenic disturbances such as partial cutting can increase the complexity of forest patterns as measured by digital photos and that the intermediate disturbance hypothesis could be applied to the observation of complexity.

Most interestingly, we tested a new index to measure the complexity of forest patterns in digital photographs that accounts for the patterns created by forest objects and light in forests. Although this index could be further adjusted and tested to facilitate its interpretation, its efficiency for the evaluation of the effects of disturbances is promising.

\section{References}

Aakala, T., S. Fraver, B.J. Palik and A.W. D'Amato. 2012. Spatially random mortality in old-growth red pine forests of northern Minnesota. Canadian Journal of Forest Research 42(5): 899-907.

Aguilar-Amuchastegui, N. and G.M. Henebry. 2007. Assessing sustainability indicators for tropical forests: Spatio-temporal heterogeneity, logging intensity, and dung beetle communities. Forest Ecology and Management 253(1-3): 56-67.

Andrienko, Y.A., N.V. Brilliantov and J. Kurths. 2000. Complexity of two-dimensional patterns. European Physical Journal B 15(3): 539-546. 
Barbosa Lima, A., J.J. Corral Rivas, K. Von Gadow and U. Muuss. 2006. Spatial Structure Analysis Using Three Different Approaches. In Proceedings, Tropentag 2006. International Research on Food Security, Natural Resource Management and Rural Development, University of Bonn, October 11-13, 2006. Available at http://www.tropentag. de/2006/abstracts/full/375.pdf.

Beaudet, M. and C. Messier. 2002. Variation in canopy openness and light transmission following selection cutting in northern hardwood stands: an assessment based on hemispherical photographs. Agricultural and Forest Meteorology 110(3): 217-228.

Beaudet, M., C. Messier and A. Leduc. 2004. Understory light profiles in temperate deciduous forests: Recovery process following selection cutting. Journal of Ecology 92(2): 328-338.

Brais, S., B.D. Harvey, Y. Bergeron, C. Messier, D. Greene, A. Belleau and D. Paré. 2004. Testing forest ecosystem management in boreal mixedwoods of northwestern Quebec: initial response of aspen stands to different levels of harvesting. Canadian Journal of Forest Research 34(2): 431-446.

Connell, J.H. 1978. Diversity in Tropical Rain Forests and Coral Reefs. Science 199(4335): 1302-1310.

Crow, T.R., D.S. Buckley, E.A. Nauertz and J.C. Zasada. 2002. Effects of management on the composition and structure of northern hardwood forests in Upper Michigan. Forest Science 48(1): 129-145.

Dale, M.B., M. Anand and R.E. Desrochers. 2007. Measuring information-based complexity across scales using cluster analysis. Ecological Informatics 2(2): 121-127.

Denslow, J.S. 1985. Disturbance-mediated coexistence of species. In S.T.A. Pickett and P.S. White (eds.). Ecology of Natural Disturbance and Patch Dynamics. pp. 307-323. Academic Press, Orlando, FL.

Denslow, J.S. and S. Guzman. 2000. Variation in Stand Structure, Light and Seedling Abundance across a Tropical Moist Forest Chronosequence, Panama. Journal of Vegetation Science 11(2): 201-212.

Drever, C.R., G. Peterson, C. Messier, Y. Bergeron and M. Flannigan. 2006. Can forest management based on natural disturbances maintain ecological resilience? Canadian Journal of Forest Research 36(9): 2285-2299.

Endler, J.A. 1993. The Color of Light in Forests and Its Implications. Ecological Monographs 63(1): 2-27.

Grabarnik, P. and A. Särkkä. 2009. Modelling the spatial structure of forest stands by multivariate point processes with hierarchical interactions. Ecological Modelling 220(9-10): 1232-1240.

Gunderson, L.H. 2000. Ecological Resilience - In Theory and Application. Annual Review of Ecology and Systematics 31: 425-439.

Hagerhall, C.M., T. Purcell and R. Taylor. 2004. Fractal dimension of landscape silhouette outlines as a predictor of landscape preference. Journal of Environmental Psychology 24(2): 247-255.

Heinimann, H.R. 2010. A concept in adaptive ecosystem management - An engineering perspective. Forest Ecology and Management 259(4): 848-856.

Holling, C.S. 2004. From Complex Regions to Complex Worlds. Ecology and Society 9(1): 11 .

Kane, V.R., R.F. Gersonde, J.A. Lutz, R.J. McGaughey, J.D. Bakker and J.F. Franklin. 2011. Patch dynamics and the development of structural and spatial heterogeneity in Pacific Northwest forests. Canadian Journal of Forest Research 41(12): 2276-2291.

Kretzschmar, A., S. Soubeyrand and N. Desassis. 2010. Aggregation patterns in hierarchy/proximity spaces. Ecological Complexity 7(1): 21-31.

Li, M.P. and M.B. Vitányi. 1994. An introduction to Kolmogorov complexity and its applications. Springer Verlag, Berlin. 546 p.

Loehle, C. et al. 2005. Managed forest landscape structure and avian species richness in the southeastern US. Forest Ecology and Management 214(1-3): 279-293.

Marsden, S.J., A.H. Fielding, C. Mead and M.Z. Hussin. 2002. A technique for measuring the density and complexity of understory vegetation in tropical forests. Forest Ecology and Management 165(13): 117-123.

Mazerolle, M.J. 2011. AICcmodavg: Model selection and multimodel inference based on (Q)AIC(c). R package version 1.17.
McElhinny, C., P. Gibbons, C. Brack and J. Bauhus. 2005. Forest and woodland stand structural complexity: Its definition and measurement. Forest Ecology and Management 218(1-3): 1-24.

McGee, G.G., D.J. Leopold and R.D. Nyland. 1999. Structural characteristics of old-growth, maturing, and partially-cut northern hardwood forests. Ecological Applications 9(4): 1316-1329.

Messier C., K.J. Puettmann and D.K. Coates. 2013. Managing Forests as Complex Adaptive Systems - Building Resilience to the Challenge of Global Change. Routledge. 189 p.

Messier, C., R. Tittler, D.D. Kneeshaw, N. Gélinas, A. Paquette, K. Berninger, H. Rheault, P. Meek and N. Beaulieu. 2009. TRIAD zoning in Quebec: Experiences and results after 5 years. The Forestry Chronicle 85(6): 885-896.

Neumann, M. and F. Starlinger. 2001. The significance of different indices for stand structure and diversity in forests. Forest Ecology and Management 145(1-2): 91-106.

Parrott, L. 2005. Quantifying the complexity of simulated spatiotemporal population dynamics. Ecological Complexity 2(2): 175-184.

Pinheiro, J., D. Bates, S. DebRoy, D. Sarkar and R Development Core Team. 2011. nlme: Linear and Nonlinear Mixed Effects Models. R package version 3.1-98.

Pommerening, A. 2006. Evaluating structural indices by reversing forest structural analysis. Forest Ecology and Management 224(3): 266-277. Proulx, R. and L. Parrott. 2008. Measures of structural complexity in digital images for monitoring the ecological signature of an old-growth forest ecosystem. Ecological Indicators 8(3): 270-284.

Puettmann, K., Coates, K.D. and Messier, C. 2009. A critique of silviculture: managing for complexity. Island Press, Washington, DC. 189 p. R Development Core Team. 2010. R: A Language and Environment for Statistical Computing. R Foundation for Statistical Computing, Vienna. 409 p.

Roberts, M.R. 2007. A conceptual model to characterize disturbance severity in forest harvests. Forest Ecology and Management 242(1): 58-64. Saunders, M.R. and R.G. Wagner. 2008. Long-term spatial and structural dynamics in Acadian mixedwood stands managed under various silvicultural systems. Canadian Journal of Forest Research 38(3): 498-517. Smith, A.R. 1978. Color Gamut Transform Pairs. In SIGGRAPH 78 Conference Proceedings, Aug. 12-19, 1978. Reprinted in J.C. Beatty and K.S. Booth. Tutorial, Computer Graphics 2nd Ed., 1982. pp. 376-383. IEEE Computer Society Press, Silver Spring, MD. Available at alvyray.com/Papers/CG/color78.pdf.

Staudhammer, C.L. and V.M. LeMay. 2001. Introduction and evaluation of possible indices of stand structural diversity. Canadian Journal of Forest Research 31(7): 1105-1115.

Sugihara, G. and R.M. May. 1990. Applications of fractals in ecology. Trends in Ecology and Evolution 5(3): 79-86.

Suzuki, N. and J.P. Hayes. 2003. Effects of thinning on small mammals in Oregon coastal forests. Journal of Wildlife Management 67(2): 352-371.

Tittler, R., C. Messier and P.J. Burton. 2001. Hierarchical forest management planning and sustainable forest management in the boreal forest. The Forestry Chronicle 77(6): 998-1005.

Turner, M.G. 1989. Landscape ecology: the effect of pattern on process. Annual review of ecology and systematics 20: 171-197.

Van Den Meersschaut, D. and K. Vandekerkhove. 1998. Development of a stand-scale forest biodiversity index based on the State Forest Inventory. In M. Hansen and T. Burk (eds.). Proceedings, Boise Centre on the Grove, Boise, ID, August 16-20, 1998. pp. 340-349. Available at http://www.nrs.fs.fed.us/pubs/276.

Vepakomma, U., B. St-Onge and D.D. Kneeshaw. 2008. Spatially explicit characterization of boreal forest gap dynamics using multi-temporal lidar data. Remote Sensing of Environment 112(5): 2326-2340.

Wiegand, T., W.D. Kissling, P.A. Cipriotti and M.R. Aguiar. 2006. Extending point pattern analysis for objects of finite size and irregular shape. Journal of Ecology 94(4): 825-837.

Zenner, E.K. and D.E. Hibbs. 2000. A new method for modelling the heterogeneity of forest structure. Forest Ecology and Management 129(1-3): 75-87. 